\title{
Percepción del clima laboral de docentes y funcionarios de una Institución Educativa Municipal de la Ciudad de San Juan de Pasto
}

\author{
Sonia Maritza \\ Matabanchoy Tulcan \\ PhD. Psicología \\ Universidad de Nariño, Colombia \\ Correo electrónico: somapsicologa@yahoo.es
}

Jose Luis Chaucanes Guerrero

Psicólogo

Universidad de Nariño, Colombia

Correo electrónico: joseluischaucanes@gmail.com
Recibido: 29/05/2019

Evaluado: 28/08/2019

Aceptado: 03/09/2019

\section{Resumen}

El presente estudio evalúa la percepción del clima organizacional en docentes y funcionarios pertenecientes a una Institución Educativa Municipal en San Juan de Pasto mediante un diseño de tipo cuantitativo descriptivo con corte transversal, para ello se empleó el Instrumento del Departamento Administrativo de la Función Pública DAFP con una muestra conformada por 204 funcionarios entre docentes y directivos. Los resultados muestran que las variables Administración del Talento Humano y Medio Ambiente Físico se presentan como aspectos por mejorar; por otro lado, los factores que se presentan como fortalezas son: la Capacidad Profesional seguida por Trabajo en grupo y Estilo de Dirección. Se concluye que conocer las fortalezas y debilidades que hacen parte del clima de la organización es indispensable para generar estrategias de prevención e intervención, así como también potenciar y optimizar aquellos elementos que influyen de manera positiva con el fin de que favorezcan al bienestar de los funcionarios mejorando el clima organizacional y la calidad educativa.

Palabras clave DAFP, Evaluación de clima organizacional, Institución educativa.

1 Para citar este artículo: Matabanchoy, S. y Chaucanes, J. (2021). Percepción del clima laboral de docentes y funcionarios de una Institución Educativa Municipal de la Ciudad de San Juan de Pasto. Informes Psicológicos, 21(2), pp.13-26 http://dx.doi.org/10.18566/infpsic.v21n2a01 


\section{Perception of the work environment of teachers and officials of a Municipal Educational Institution in the City of San Juan de Pasto}

Abstract

This study assesses the perception of the organizational climate in teachers and officials belonging to a Municipal Educational Institution in San Juan de Pasto. It is carried out through a descriptive quantitative crosssectional design, for which the Instrument of the Administrative Department of Public Function DAFP was used with a sample made up of 204 officials between teachers and managers. The results show that the variables Human Talent Management and Physical Environment are presented as aspects to improve. On the other hand, the factors that are presented as strengths are: Professional Capacity followed by Group Work and Management Style. It is concluded that knowing the strengths and weaknesses that are part of the organization's climate is essential to generate prevention and intervention strategies, as well as to promote and optimize those elements that have a positive influence in order to favor the well-being of officials and to improve the organizational climate and educational quality.

\section{Percepção do ambiente de trabalho de professores e funcionários de uma Instituição Municipal de Ensino da Cidade de San Juan de Pasto}

Resumo

0 presente estudo avalia a percepção do clima organizacional em professores e funcionários pertencentes a uma Instituição Educacional Municipal de San Juan de Pasto por meio de um desenho quantitativo descritivo com corte transversal, para isso o Instrumento Administrativo do Departamento de Função Pública DAFP foi usado com uma amostra composta por 204 funcionários entre professores e gestores. Os resultados mostram que as variáveis Gestão do Talento Humano e Ambiente Físico se apresentam como aspectos a serem melhorados. Por outro lado, os fatores que se apresentam como pontos fortes são: Capacidade Profissional seguida de Trabalho em Grupo e Estilo de Gestão. Conclui-se que conhecer os pontos fortes e fracos que fazem parte do clima da organização é essencial para gerar estratégias de prevenção e intervenção, bem como potencializar e otimizar aqueles elementos que influem positivamente para favorecer o bem-estar dos funcionários e geram melhoras no clima organizacional e na qualidade educacional. 


\section{ntroducción}

El concepto de Clima Organizacional aparece por primera vez en el campo de la psicología organizacional gracias a Saúl William Gellerman en 1960 y parte del hecho de que la interacción entre el hombre y el ambiente genera diversos comportamientos que afectan el funcionamiento de sistemas organizados en grupos o colectividades, por lo tanto, influyen en el ambiente de la organización (Segredo, 2013). Por otra parte, Velez-Alvarado y Gonzáles (2019) lo describen como las expectativas y demandas que tienen los trabajadores de una institución en torno a ella, incluyendo su nivel de satisfacción frente al funcionamiento de su lugar de trabajo.

Noriega (2011) afirma que el clima organizacional es un constructo complejo enmarcado en dos enfoques, el primero se denomina estructuralista, que resalta los factores organizacionales meramente objetivos y, el subjetivista o perceptual, que destaca las condiciones que perciben los individuos dentro de la organización. La importancia de este último está en el hecho de que los comportamientos de un colaborador no son consecuencia de los factores propios de la organización, sino que depende de la percepción que tiene el trabajador sobre ellos; sin embargo, esta percepción depende de las experiencias que cada individuo tenga con su organización, de ahí que el clima laboral sea reflejo de la interacción entre las características de la persona y la organización.

Para una organización resulta importante conocer y medir las percepciones del clima organizacional, ya que éste puede impactar de manera significativa en los resultados (Iglesias \& Sánchez, 2015); existen diversos factores de riesgo que pueden afectar al trabajador, ya sea en su desempeño laboral o en su vida personal, dificultando los procesos y el buen funcionamiento de la organización y se hace necesario conocerlos e identificarlos. Después de realizar un diagnóstico del clima organizacional se logra determinar la percepción que tienen los colaboradores acerca del mismo y ofrecer información relevante sobre su potencial productividad; además, establece en qué medida le pueden afectar los factores internos y externos a la propia organización y de qué forma influye en su capacidad para lograr sus objetivos. Con lo anterior, se puede decir que el clima organizacional tiene gran importancia, dado que refleja la cultura organizacional, la cual es adoptada por los funcionarios y es de ellos de quien dependerá la eficacia y productividad de la organización (Peña, 2017).

Cada organización es diferente y es por lo anterior que en las instituciones educativas también se considera importante evaluar el clima organizacional pues trabajar diariamente en dichas organizaciones implica una interacción entre docentes, administrativos y directivos, además de la convivencia con sus estudiantes, un trabajo de equipo, a veces rutinario, estresante en otras ocasiones la interacción resulta inadecuada y dificulta los procesos de comunicación, incidiendo directamente en su percepción de bienestar y en su desempeño dentro de la institución. Por ello es muy necesario hacer un seguimiento, una medición y evaluación que permita tener un panorama claro y así conocer el estado del clima organizacional y de esta forma se logre optimizar los recursos y el talento 
humano con el fin de aportar en el desarrollo de los objetivos propuestos (Herazo, Ospina \& Ramírez, 2013).

En las instituciones educativas municipales el clima organizacional es un proceso que aún requiere de precisión, definición, organización, seguimiento constante y medición; de tal manera que se sienten las bases para que los trabajadores se desempeñen diligentemente para alcanzar los objetivos propuestos por la organización buscando la excelencia, calidad e integridad. Lográndose a través de la evaluación de desempeño, la constante capacitación del talento humano y el empleo de técnicas de resolución conflictos (Peña, 2017).

El clima organizacional puede marcar la diferencia entre una organización productiva y otra que no lo es, por esto, se hace necesario que dentro de las empresas se busque establecer relaciones laborales sanas y armoniosas e implantar un sistema de retroalimentación que permita orientar y coordinar acciones. Para desarrollar estas estrategias se deben adelantar actividades encaminadas a la medición frecuente del clima de la organización, a fin de conocer la percepción de los funcionarios con respecto a éste, e instaurar acciones que mejoren su motivación y, por ende, el bienestar en la entidad (Chacón, Quiñones \& Vigoya, 2005).

Por lo tanto, se resalta la importancia de conocer la percepción que tiene el talento humano frente al ambiente laboral en la organización, en vista de que se sabe que el desempeño de los trabajadores se rige por las percepciones acerca de sus características personales y las condiciones en las que se encuentra laborando; de ahí que cuando existe pérdida de motivación, insatisfacción y absentismo, se presenta un gran impacto en la eficiencia y la eficacia de la organización, lo cual puede generar un clima laboral inadecuado y poner en riesgo el éxito de cualquier estrategia de mejora para conseguir los objetivos de la organización (Williams, 2013).

La revisión de literatura muestra que regionalmente no se han llevado a cabo estudios acerca del clima organizacional en instituciones educativas; por lo anterior, el objetivo general de este estudio fue comprender las percepciones del clima organizacional en colaboradores pertenecientes a una institución educativa municipal a través del Instrumento del Departamento Administrativo de la Función Pública DAFP para medir clima laboral.

Es importante resaltar que, aunque existen diversos instrumentos y metodologías para la medición del clima organizacional, generalmente se hace a través de encuestas aplicadas a los colaboradores de una organización o a un área dentro de la misma que se desee evaluar (Iglesias \& Sánchez, 2015).

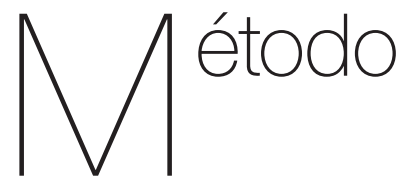

\section{Diseño}

La investigación se realizó desde el paradigma cuantitativo puesto que el conocimiento obtenido es resultado de la observación, medición e interpretación minuciosa de la realidad. De tipo descriptivo, en vista de que se pretende observar un fenómeno desconocido y describirlo objetivamente sin que exista manipulación 
de variables. Y, finalmente, de corte transversal, ya que la observación se realiza en un solo punto en el tiempo y se analiza la relación entre las variables (Sousa, Driessnack \& Mendes, 2007).

\section{Participantes}

La muestra estuvo conformada por 204 funcionarios de una institución educativa municipal, entre docentes, directivos y personal administrativo, con edades entre los 25 y 65 , de los cuales 131 pertenecen a la sede central o bachillerato y 73 a la sede primaria, con un 65\% de mujeres y un 35\% de hombres. Se realizó muestreo no probabilístico por conveniencia, no se tuvieron en cuenta criterios de inclusión ya que este tipo de muestreo permite seleccionar sujetos que, por su proximidad y accesibilidad, acepten participar y ser incluidos en la investigación (Otzen \& Manterola, 2017).

\section{Técnicas e instrumentos}

En la medición de clima laboral en la Institución Educativa Municipal se empleó el Instrumento del Departamento Administrativo de la Función Pública DAFP para evaluar clima laboral, diseñado en Colombia. El instrumento fue construido de acuerdo con la metodología ofrecida por Rensis Likert, la cual ha mostrado ser adecuada para medir actitudes que tienen las personas frente a determinados fenómenos sociales. Consta de 51 ítems validados estadísticamente a través de la prueba chi-cuadrado $X^{2}$ y a través de la estrategia de validación por expertos. El análisis factorial indica evidencias de validación de contenido y una consistencia interna de $\alpha=.94$. Los ítems se encuentran organizados en siete variables las cuales se presentan a continuación:

Tabla 1.

Variables que conforman el DAFP

\begin{tabular}{ll}
\hline Variable & Definición \\
\hline Orientación organizacional & $\begin{array}{l}\text { Claridad de los trabajadores con respecto a la misión, los objetivos, valores y políticas de una institución, y } \\
\text { de la forma como se realiza la planeación y los procesos, se asignan las funciones y se brindan los recursos } \\
\text { necesarios para cumplir efectivamente con su labor. }\end{array}$ \\
Administración del talento humano & $\begin{array}{l}\text { Hace referencia a la percepción que tienen los trabajadores acerca de los procesos organizacionales enca- } \\
\text { minados a ubicar adecuadamente a los funcionarios en sus respectivos cargos, a su satisfacción, bienestar y } \\
\text { capacitación, en pro de su crecimiento profesional y personal. }\end{array}$ \\
Estilo de dirección & $\begin{array}{l}\text { Se relaciona con las habilidades y conocimientos gerenciales aplicados en el desempeño de las funciones } \\
\text { del área; destrezas y rasgos personales para orientar a los colaboradores en la consecución de los objetivos } \\
\text { de la organización. }\end{array}$ \\
Comunicación e integración & $\begin{array}{l}\text { Intercambio de ideas, sentimientos y pensamientos entre dos o más personas por medio del lenguaje oral, } \\
\text { escrito o mímico; orientado a fortalecer la afinidad y la unión entre los trabajadores de una entidad. }\end{array}$ \\
Trabajo en grupo & Es el que realiza un determinado número de personas que trabajan juntos aportando sus habilidades para \\
lograr un propósito común con el cual se comprometen y responsabilizan. \\
Capacidad profesional
\end{tabular}

Fuente: Manual del Instrumento del Departamento Administrativo de la Función Pública (DAFP) 


\section{Procedimiento}

La presente investigación se desarrolló en varias fases, iniciando con la fase preparatoria en la cual se eligió el tema a estudiar, se planificaron las actividades que se realizaron en las fases posteriores y se seleccionó el instrumento para la recolección de datos; en segunda instancia se llevó a cabo la fase de trabajo de campo, en la que se realizó un proceso de información y sensibilización hacia los funcionarios, y el diligenciamiento del instrumento, que se realizó en un espacio físico adecuado dentro de las instalaciones de la Institución Educativa. En la fase de desarrollo productivo de datos se tomó lo más esencial de la información según el objetivo propuesto y se realizó el proceso de vaciado de información en una base de datos, seguidamente, se procedió con el análisis de los datos obtenidos mediante el programa estadístico IBM SPSS Statistics. En la fase Informativa se elaboró un informe final en donde se consignaron los resultados de la aplicación del instrumento $y$, finalmente, se llevó a cabo la fase de devolución de emergentes, en ésta etapa se entregó un informe escrito y verbal a los participantes sobre los resultados encontrados.

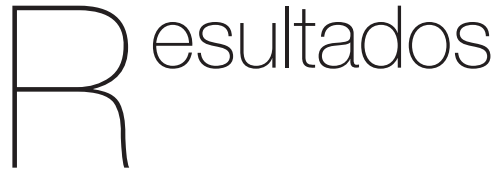

A partir de la aplicación del instrumento, se describen los resultados obtenidos en cuanto a cada una de sus variables con el fin de obtener un diagnóstico que evidencie la calidad del clima organizacional dentro de la institución.

Es importante resaltar que de acuerdo con la revisión del manual del Instrumento del Departamento Administrativo de la Función Pública (DAFP) una variable se considera favorable cuando el porcentaje de la suma del total de respuestas de cada ítem marcado como "acuerdo y total de acuerdo" se acerca a 100\%, evidenciando una fortaleza en el clima organizacional, y la suma de los porcentajes de los ítems marcados como "desacuerdo y total desacuerdo" representan una percepción desfavorable cuando tiende a 50\% (Chacón, Quiñonez \& Vigoya, 2004).

Considerando lo mencionado anteriormente, a continuación, se muestran los resultados obtenidos tanto en la sede central o de bachillerato y la sede primaria.

Tabla 2.

Variable Orientación Organizacional

\begin{tabular}{llcc}
\hline \multirow{2}{*}{ Orientación Organizacional } & & \multicolumn{2}{c}{ Sede } \\
\cline { 3 - 4 } Total Desacuerdo & Recuento & Central & Primaria \\
\hline \multirow{2}{*}{ Desacuerdo } & $\%$ dentro de Sede & 127 & 36 \\
& Recuento & $9.4 \%$ & $7.9 \%$ \\
\hline \multirow{2}{*}{ Acuerdo } & $\%$ dentro de Sede & 168 & 54 \\
& Recuento & $12.4 \%$ & $11.8 \%$ \\
\hline \multirow{2}{*}{ Total Acuerdo } & $\%$ dentro de Sede & 530 & 200 \\
& Recuento & $39.0 \%$ & $43.9 \%$ \\
\hline
\end{tabular}

Fuente: Creación propia 
La variable denominada Orientación en bachillerato y $80 \%$ en primaria la apreOrganizacional se percibe como una for- cian positivamente. taleza en la institución puesto que el 78\%

Tabla 3.

Variable Administración del talento humano

\begin{tabular}{llcc}
\hline \multirow{2}{*}{ Administración del Talento Humano } & \multicolumn{2}{c}{ Sede } \\
\cline { 2 - 3 } Total Desacuerdo & Recuento & Central & Primaria \\
& $\%$ dentro de Sede & 117 & 19 \\
\hline \multirow{2}{*}{ Desacuerdo } & Recuento & $13.1 \%$ & $6.5 \%$ \\
\hline \multirow{2}{*}{ Acuerdo } & $\%$ dentro de Sede & 225 & 56 \\
& Recuento & $25.2 \%$ & $19.2 \%$ \\
\hline \multirow{2}{*}{ Total Acuerdo } & $\%$ dentro de Sede & 331 & 145 \\
& Recuento & $37.1 \%$ & $49.8 \%$ \\
\hline
\end{tabular}

Fuente: Creación propia

Esta variable presenta un porcentaje positivo mayor en las sedes de primaria (74\%) que en la sede central o de bachillerato (62\%), por lo tanto, la primera representa una fortaleza con respecto a la segunda ya que el porcentaje de percepción negativa en ésta es de 38\%.

Tabla 4.

Variable Estilo de Dirección

\begin{tabular}{llcc}
\hline \multirow{2}{*}{ Estilo de Dirección } & & \multicolumn{2}{c}{ Sede } \\
\cline { 3 - 4 } Total Desacuerdo & Recuento & Central & Primaria \\
& \% dentro de Sede & 156 & 28 \\
\hline \multirow{2}{*}{ Desacuerdo } & Recuento & $10.4 \%$ & $5.7 \%$ \\
\hline \multirow{2}{*}{ Acuerdo } & \% dentro de Sede & 182 & 29 \\
& Recuento & $12.1 \%$ & $5.9 \%$ \\
\hline \multirow{2}{*}{ Total Acuerdo } & $\%$ dentro de Sede & 647 & 271 \\
& Recuento & $43.0 \%$ & $55.4 \%$ \\
\hline
\end{tabular}

Fuente: Creación propia

El $77 \%$ de los participantes de la sede central y el $88 \%$ en las sedes de primaria reconocen esta variable como una gran fortaleza del clima laboral de la organización. La percepción negativa encontrada en esta variable es del 23\% y $12 \%$ en la sede central y sedes primarias respectivamente. 
Tabla 5.

Variable Comunicación e Integración

\begin{tabular}{llcc}
\hline \multirow{2}{*}{ Comunicación e Integración } & & \multicolumn{2}{c}{ Sede } \\
\cline { 2 - 3 } Total Desacuerdo & Recuento & Central & Primaria \\
\hline \multirow{2}{*}{ Desacuerdo } & $\%$ dentro de Sede & 91 & 23 \\
& Recuento & $10.1 \%$ & $7.8 \%$ \\
\hline \multirow{2}{*}{ Acuerdo } & $\%$ dentro de Sede & 159 & 37 \\
& Recuento & $17.6 \%$ & $12.5 \%$ \\
\hline \multirow{2}{*}{ Total Acuerdo } & $\%$ dentro de Sede & 381 & 156 \\
& Recuento & $42.2 \%$ & $52.9 \%$ \\
\hline
\end{tabular}

Fuente: Creación propia

La variable denominada Comunica- en la sede primaria, con un $72 \%$ y $80 \%$ ción e integración es valorada positiva- respectivamente.

mente, tanto en la sede bachillerato como

Tabla 6.

Variable Trabajo en Grupo

\begin{tabular}{llcc}
\hline \multirow{2}{*}{ Trabajo en Grupo } & & \multicolumn{2}{c}{ Sede } \\
\cline { 3 - 3 } Total Desacuerdo & & Central & Primaria \\
& Recuento & 33 & 11 \\
Desacuerdo & Recuento & $4.4 \%$ & $4.5 \%$ \\
\hline \multirow{2}{*}{ Acuerdo } & $\%$ dentro de Sede & 84 & 24 \\
& Recuento & $11.2 \%$ & $9.8 \%$ \\
\hline \multirow{2}{*}{ Total Acuerdo } & $\%$ dentro de Sede & 380 & 152 \\
& Recuento & $50.8 \%$ & $61.8 \%$ \\
\hline
\end{tabular}

Fuente: Creación propia

En cuanto a la variable de Trabajo en Grupo, un $84 \%$ en la sede central y un $86 \%$ de los participantes en las sedes primarias tienen una visión positiva de este factor. El porcentaje de percepción negativo es significativamente bajo con un $16 \%$ en la sede central y un $14 \%$ en las sedes primarias. 
Tabla 7.

Variable Capacidad profesional

\begin{tabular}{llcc}
\hline \multirow{2}{*}{ Capacidad Profesional } & & \multicolumn{2}{c}{ Sede } \\
\cline { 3 - 4 } Total Desacuerdo & Recuento & Central & Primaria \\
\hline \multirow{2}{*}{ Desacuerdo } & \% dentro de Sede & 98 & 30 \\
& Recuento & $8.2 \%$ & $7.6 \%$ \\
\hline Acuerdo & \% dentro de Sede & 53 & 14 \\
\hline \multirow{2}{*}{ Total Acuerdo } & Recuento & $4.4 \%$ & $3.5 \%$ \\
\hline
\end{tabular}

Fuente: Creación propia

Los funcionarios de la Institución Edu- sede central y un 89\% en las primarias. cativa perciben como fortaleza de su cli- $\quad$ La percepción negativa en esta variable ma laboral, principalmente, la variable de corresponde al $12 \%$ en la sede central y capacidad profesional con un $88 \%$ en la $11 \%$ en las sedes primarias.

Tabla 8.

Variable Medio Ambiente Físico

\begin{tabular}{llcc}
\hline \multirow{2}{*}{ Medio Ambiente Físico } & & \multicolumn{2}{c}{ Sede } \\
\cline { 3 - 4 } Total Desacuerdo & Recuento & Central & Primaria \\
\hline \multirow{2}{*}{ Desacuerdo } & \% dentro de Sede & 125 & 55 \\
& Recuento & $11.9 \%$ & $15.8 \%$ \\
\hline Acuerdo & \% dentro de Sede & 287 & 58 \\
\hline & Recuento & $27.2 \%$ & $16.7 \%$ \\
\hline Total Acuerdo & $\%$ dentro de Sede & 431 & 150 \\
& Recuento & $40.9 \%$ & $43.1 \%$ \\
\hline
\end{tabular}

Fuente: Creación propia

La variable medio ambiente físico muestra que el 39\% en la sede central y el $31 \%$ en las sedes primarias consideran esta variable como un factor de riesgo, siendo estos los valores negativos más altos. 


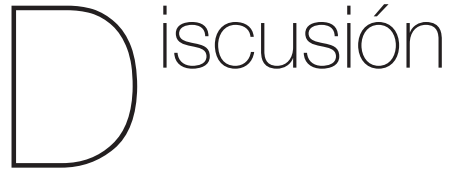

Actualmente se reconoce que un adecuado clima laboral brinda las condiciones óptimas y necesarias para que los trabajadores de una organización se sientan motivados y puedan cumplir con las tareas de forma eficiente, lo que influye en gran medida tanto en la productividad como en el desarrollo del talento humano (Ramos, 2012), y puede comprenderse como la percepción que los trabajadores tienen en común sobre las políticas y prácticas que se ejercen en la institución (Marín, Dos Santos, Raad, Ávila, \& Siqueria, 2014) que se presentan como normas y pautas de comportamiento dentro de la organización (Cuadra \& Veloso, 2007).

El Ministerio de Trabajo establece que se debe implementar el Sistema de Gestión de Seguridad y Salud en el Trabajo SG-SST, proceso que se fundamenta en la mejora constante de la organización cuyo objetivo es anticipar, reconocer, evaluar y controlar aquellos riesgos que pudieran afectar en alguna medida al trabajador, destacando, entre ellos, el entorno físico como un factor importante. En esta Institución Educativa, tanto en las sedes de bachillerato como en primaria, los colaboradores perciben que el medio ambiente físico de la organización incide negativamente en su ambiente laboral, y, en consecuencia, en el clima organizacional. Tener buena iluminación, estímulos visuales y auditivos, seguridad, espacio, entre otros factores, afecta positivamente el bienestar del trabajador, reflejándose en su ánimo y confort, convirtiendo al ambiente físico en un factor decisivo del clima laboral (Chacón et al., 2005). Así pues, es importante implementar el SG-SST debido a que mejora el ambiente del trabajo, el bienestar del individuo y la calidad de vida laboral, reduce el ausentismo y la accidentalidad, influyendo positivamente, de esta manera, en el clima laboral, y, por ende, en la productividad (Ministerio del Trabajo, 2015).

Dentro del clima laboral, también se encuentra la variable de administración de talento humano, que hace referencia a la capacidad de gestionar adecuadamente el recurso humano por parte de los mandos inmediatos; además se relaciona con el apoyo social de superiores, teniendo en cuenta que éste representa el funcionamiento de las relaciones entre los trabajadores, lo que podría afectar positiva o negativamente a la salud del trabajador (Chicaiza, 2014). En la institución existe una percepción negativa hacia los aspectos que conforman esta variable, por lo tanto, se hace necesario intervenir en este factor buscando reducir el riesgo de posibles dificultades en el bienestar del trabajador, aumentando las posibilidades de éxito de la organización, optimizando y mejorando la calidad del trabajo, la producción, la motivación y el compromiso con su institución. Se considera importante evaluar este factor en la institución educativa puesto que una evaluación de este proceso permitiría reconcer y comunicar a los trabajadores la manera como desempeñan su trabajo y, simultáneamente, cumplir un plan de mejora continua; además, se logra identificar aquellas personas que necesitan entrenamiento, cambio de puesto, un ascenso o ser separados de la institución (Macías, Romero \& Verduga, 2015). 
Según Chacón et al. (2005), otro aspecto que influye en el clima laboral es el trabajo en grupo, siendo, para los funcionarios de la Institución Educativa, una variable que fortalece en gran medida el clima que se percibe en la organización. En este sentido, Aguilar (2016) afirma que las organizaciones requieren de trabajo en equipo pues el éxito de ellas depende de la cohesión, la comunicación y el compromiso de quienes la conforman. Cuando los trabajadores comparten los mismos objetivos de la organización el clima se ve favorecido, incrementando la productividad, mejorando la comunicación y cumpliendo las tareas complejas con mayor éxito; además, favorece la integración, satisfacción, creatividad, seguridad, desarrollo personal y profesional de todos los miembros, mientras que un clima laboral inadecuado infiere una falta de identificación con sus objetivos, así como un posible deterioro del ambiente de trabajo. En las sedes de primaria y bachillerato de la institución educativa, los colaboradores perciben como una fortaleza la variable de trabajo en grupo, pues reconocen que existe una adecuada cohesión entre directivos, administrativos y docentes y, como se mencionó anteriormente, un adecuado trabajo en grupo fortalece el clima laboral, pues, al trabajar conjuntamente para alcanzar los objetivos que la institución se plantea, se logra que las relaciones entre compañeros y con la organización esté satisfecha.

Se ha comprobado que las bases de un buen clima laboral se asocian, en términos generales, con la productividad y buen desempeño de la organización y, más específicamente, con factores como la calidad directiva, entre otros (Cuadra \& Veloso, 2007). El estilo de dirección es un factor que tiende a provocar efectos importantes en los trabajadores, así pues, los directivos que demuestran un estilo de dirección adecuado contribuyen a generar resultados positivos sobre la salud de los empleados que, a su vez, tiene un impacto en su productividad; por otro lado, un estilo de dirección inadecuado impactaría de forma negativa tanto en el bienestar como en el desempeño dentro de la institución (Soler, 2008). En vista de que la competencia de los dirigentes repercute directamente en el éxito de las organizaciones (Ramos, 2012) el estilo de dirección es una variable que con una adecuada gestión puede tener efectos significativos en el funcionamiento de la organización y se debería continuar potencializando con el fin de reducir la percepción negativa de algunos de sus trabajadores, ya que en la institución se presenta como una fortaleza que, por lo tanto, aporta en gran medida a un clima laboral favorable.

Hoy en día se sabe que las organizaciones buscan que sus colaboradores estén alineados con sus objetivos empresariales; sin embargo, actualmente son muy pocas las organizaciones que le dan importancia a las habilidades individuales de los trabajadores, las cuales son fundamentales no solo para el éxito en el desempeño de los colaboradores sino también en el éxito y resultados de la organización en la que trabajan, siendo esto un beneficio para las dos partes (Becerra \& Campos, 2012). Esta situación se ve reflejada por los colaboradores de la institución, pues perciben que sus habilidades y capacidades personales son fundamentales en el momento de trabajar en pro de los objetivos de su organización $y$, a la vez, les permite seguir fortaleciendo sus habilidades, para finalmente generar un adecuado desempeño en su cargo. 


\section{Conclusiones y recomendaciones}

El clima organizacional en instituciones educativas es un campo de investigación poco abordado tanto a nivel regional como nacional; se considera importante estudiarlo en este tipo de organizaciones puesto que la educación y formación de estudiantes son un pilar básico en la sociedad, por lo tanto, se hace necesario generar un clima adecuado para los docentes y funcionarios en pro de mejorar el bienestar laboral, la motivación y, por ende, la productividad de la institución educativa ya que, al mejorar las condiciones en las que los funcionarios desempeñan su labor, se contribuye positivamente en la calidad de la educación que brindan.

Para poder llevar a cabo el proceso de evaluación y posterior mejoramiento del clima laboral en una institución educativa se requiere una participación activa de sus directivas, puesto que son los principales responsables del rendimiento de la misma; esto se verá reflejado en la colaboración e interés por parte de docentes y demás funcionarios.

Los resultados obtenidos en el estudio pueden ser de gran ayuda para que la institución se encargue de generar alternativas de solución y estrategias de prevención e intervención que permitan un mejoramiento en el bienestar de los docentes y funcionarios influyendo positivamente en el clima organizacional, así como también potenciar la gestión del talento humano, y en general para que las instituciones educativas tengan en cuenta y se interesen en la importancia que tiene un adecuado clima laboral para el buen funcionamiento de cualquier organización. Cuando se realiza el proceso de evaluación del clima organizacional se tiene la oportunidad de vislumbrar objetivamente las fortalezas y debilidades que presenta la institución y poder actuar en consecuencia.

Durante la realización del estudio una de las limitaciones que se tuvieron fue la disponibilidad de tiempo para la aplicación del instrumento puesto que los docentes y administrativos desempeñan sus labores en distintos horarios y cuentan con poca disponibilidad.

Finalmente, se recomienda profundizar en el tema e investigar en otras instituciones educativas de la región con el fin de tener una visión más amplia y lograr una generalización de resultados.

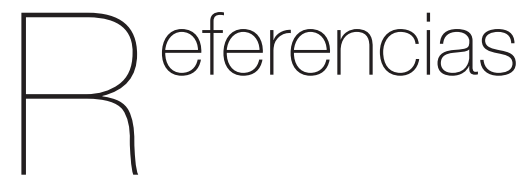

Aguado, J. (2012). Clima Organizacional de una institución educativa de ventanilla según la perspectiva de los docentes (Tesis de Maestría). Universidad San Ignacio de Loyola, Lima, Perú. Recuperado de http:// alicia.concytec.gob.pe/vufind/Record/USI L_40adOb63494ac0ae0e171bd65fce9185

Aguilar, H. (2016). Trabajo en equipo y clima organizacional (estudio realizado en colaboradores del hotel del campo de Quetzaltenango) (Tesis de Pregrado). Universidad Rafael Landívar, Quetzaltenango, Guatemala. Recuperado 
de http://recursosbiblio.url.edu.gt/ tesiseortiz/2016/05/43/Aguilar-Hector.pdf

Becerra, M. \& Campo, F. (2012). El enfoque por competencias y sus aportes en la gestión de recursos humanos (Tesis de Pregrado). Universidad de Chile, Santiago de Chile. Recuperado de http://repositorio. uchile.cl/bitstream/handle/2250/116913/ Memoria\%20MBecerra\%20FCampos. pdf?sequence $=1$

Chacón, P., Quiñónes, E. \& Vigoya, A. (2004). Guía de Intervención para la Cultura Organizacional, el Clima Laboral y el Cambio Organizacional. Recuperado de http://www.academia.edu/35591017/ Departamento_Administrativo_de_la_ Funci\%C3\%B3n_P\%C3\%BAblica

Chacón, P., Quiñónes, E. \& Vigoya, A. (2005). La calidad de vida laboral para una cultura de lo público: Instrumentos para su gestión. Recuperado de http://www1.funcionpublica.gov.co/documentacion/Componente\%20Ambiente\%20de\%20Control/ InstrumentosdeGestionparalaCalidaddeVidaLaboral.pdf

Chicaiza, B. (2014). Evaluación de los factores de riesgo psicosocial del trabajo en el centro de investigaciones de la caña de azúcar de El Ecuador (cincae) (Tesis de Pregrado). Universidad de Guayaquil, Guayaquil, Ecuador. Recuperado de http://repositorio.ug.edu.ec/bitstream/ redug/7169/1/Evaluaci\%C3\%B3n\%20 de $\% 201$ os $\% 20$ factores $\% 20$ de $\% 20$ riesgo\%20psicosocial\%20 del\%20 trabajo\%20en\%20CINCAE.pdf

Cuadra, A. \& Veloso, C. (2007). Liderazgo, Clima y Satisfacción Laboral en las Organizaciones. Universum (Talca),
22(2), 40-56. http://dx.doi.org/10.4067/ S0718-23762007000200004

Iglesias, A. \& Sánchez, Z. (2015) Generalidades del clima organizacional. Medisur, 13(3), 455-457. Recuperado de http://www. redalyc.org/articulo.oa?id=180039699016

Herazo, C., Ospina, M. \& Ramírez, S. (2013). Factores psicosociales y organizacionales que afectan la productividad (Tesis de Posgrado). Universidad de Medellín. Medellín, Colombia. Recuperado de http://repository.udem.edu. co/bitstream/handle/11407/158/ Factores\%20psicosociales\%20y\%20 organizacionales\%20que\%20afectan\%20 la\%20productividad.pdf?sequence=1

Macías, N., Romero, M. \& Verduga, A. (2015). Clima organizacional enfocado al desempeño del talento humano. ECA Sinergia, 6(1), 151-160. Recuperado de https://dialnet.unirioja.es/servlet/ articulo?codigo $=6197622$

Marín, F., Dos Santos, A., Raad, A., Ávila, A., \& Siqueria, J. (2014). Relación entre los constructos Clima Organizacional, Apoyo Laboral y Salud Organizacional en una muestra de estudiantes que trabajan. Ciencias Psicológicas, 8(1), 7-16. Recuperado de http://www.redalyc.org/ articulo.oa?id=459545412002

Ministerio del Trabajo (2015). Sistema de gestión de seguridad y salud en el trabajo. Ministerio del Trabajo de Colombia. Recuperado de http://www.mintrabajo.gov. co/relaciones-laborales/riesgos-laborales/ sistema-de-gestion-de-seguridad-y-saluden-el-trabajo

Noriega, V. (2011). Instrumento para evaluar el clima organizacional en los Grupos de 
Control de Vectores. Revista Cubana de Administración de Salud Pública, 37(2), 116122. Recuperado de http://www.redalyc. org/articulo.oa?id=21418849004

Otzen, T. \& Manterola C. (2017). Técnicas de muestreo sobre una población a estudio. International Journal of Morphology, 35(1), 227-232. http://dx.doi.org/10.4067/ S0717-95022017000100037

Peña, E. (2017). Clima Organizacional en una empresa del Estado (Tesis de Posgrado). Universidad Militar Nueva Granada, Bogotá, Colombia. Recuperado de http://repository.unimilitar. edu.co/bitstream/10654/16206/1/ Pe\%C3\%B1a\%20Salas\%20Emilcer\%20 2017.pdf

Ramos, D. (2012). El clima organizacional: definición, teoría, dimensiones y modelos de abordaje (Tesis de Pregrado). Universidad Nacional Abierta y a Distancia, Fusagasugá, Cundinamarca, Colombia. Recuperado de http://repository. unad.edu.co/bitstream/10596/2111/1/ Monografia\%20Clima\%20Organizacional. pdf

Segredo, A. (2013). Clima organizacional en la gestión del cambio para el desarrollo de la organización. Revista Cubana Salud Pública, 39(2), 385-393. Recuperado de http://www.redalyc.org/articulo. oa?id=21430556017
Soler, M. (2008). La evaluación de los factores de riesgo psicosocial del trabajo en el sector hortofrutícola: el cuestionario FAPSIHOS (Tesis de Doctorado). Universidad de Murcia, España. Recuperado de http:// www.tesisenred.net/handle/10803/11033

Sousa, V., Driessnack, M. \& Mendes, I. (2007) Revisión de diseños de investigacion resaltantes para enfermeria. Parte 1: diseños de investigacion cuantitativa. Revista latinoamericana de Enfermagem, 15(3), 502-507. https://dx.doi.org/10.1590/ S0104-11692007000300022

Vélez-Alvarado, R. \& Gonzales, R. (2019). La relación entre la espiritualidad en el ambiente laboral y el compromiso organizacional en un grupo de empleados de un municipio del área oeste de Puerto Rico. Informes Psicológicos, 19(1), 11-30. http://dx.doi.org/10.18566/infpsic.v19n1a01

Williams, L. (2013). Estudio diagnóstico de clima laboral en una dependencia pública (Tesis de Maestría). Universidad Autónoma de Nuevo León. Monterrey, México. Recuperado de http://eprints.uanl. mx/3751/1/1080256607.pdf 